\title{
A NOTE ON SPACES VIA DENSE SETS
}

\author{
R. A. MAHMOUND* AND D. A. ROSE
}

\begin{abstract}
A bstract. Some spaces have been defined depending on the concept of dense set in a given topological space $(X, \tau)$ such as: resolvable space, irresolvable space, hereditarily irresolvable space, and submaximal space. We study many of their properties and explore several relationships between these spaces and SMPC function which has been defined recently as a dual of the concept of precontinuity.
\end{abstract}

\section{Introduction}

In this paper, we will use the following notational conventions: a "space" will always mean a topological space; given a space $(X, \tau)$, for and $A \subseteq X$, we denote by Int $A$ and $C l A$ the interior of $A$ and the closure of $A$ with respect to $\tau$ respectively, and "iff" for "if and only if".

A space $(X, \tau)$ is resolvable [1] if there is a dense subset $D \subseteq X$ for which $X-D$ is also dense. A space which is not resolvable is called irresolvable. A subset of $X$ is resolvable (irresolvable) if it is resolvable (irresolvable) as a subspace. A space is hereditarily irresolvable if each of its nonempty subsets is irresolvable. Such spaces were investigated by Hewitt [2] where it was shown

Received June 1, 1992.

1980 AMS Subject classification codes. Primary: 54C10; Secondary: 54D25, 54D30.

Key words and phrases: Resolvable space, irresolvable space, hereditarily irresolvable space, submaximal space, preopen set, semi-open set, SMPC, precontinuity, preirresolute, semi-open function, Hewitt representation.

* Curresnt address: Department of Mathematics, Faculty of Education, Umm A1-Qurs University, Taif, Saudi-Arabia. 
(see also Theorem 1 of [1]) that every space $(X, \tau)$ can be expressed as a disjoint union $F \cup G$ with $F$ closed and resolvable and $G$ hereditarily irresolvable. The $F$ and $G$ are unique and $F \cup G$ is called the Hewitt representation of $X$. A space $(X, \tau)$ is said to be submaximal if each of its dense subsets are open. Clearly every submaximal space is irresolvable and in fact hereditarily irresolvable. A is called preopen [4] if $A \subseteq \operatorname{Int} C l A$, and $P O(X, \tau)$ means the collection of all preopen sets in $(X, \tau)$. For any space $(X, \tau)$ let $\tau_{P}$ be the smallest topology on $X$ containing $P O(X, \tau)$. The topology $\tau^{\alpha}[5]$ is $P O(X, \tau) \cap S O(X, \tau)$ where $A \in S O(X, \tau)$ iff $A$ is semi-open [6]. i.e. $A \subseteq C l \operatorname{Int} A$. Thus, for any space $(X, \tau), \tau \subseteq \tau^{\alpha} \subseteq P O(X, \tau) \subseteq \tau_{P}$. It is also known that $P O\left(X, \tau^{\alpha}\right)=P O(X, \tau)$ (Corollary 1 of [7]). A function $f:(X, \tau) \rightarrow(Y, \sigma)$ is said to be precontinuous [4], preirresolute [8] and strongly $M$-precontinuous [9] (SMPC) if the inverse image of each open, preopen and preopen in $(Y, \sigma)$ is preopen, preopen and open in $(X, \tau)$, respectively.

\section{On Resolvability}

Theorem 1. Each semi-open subset of a resolvable space is resolvable.

Proof. Let $A \in S O(X, \tau)$, i.e. $A \subseteq C l \operatorname{Int} A \subseteq X$ and $X$ is resolvable then Int $A$ is resolvable and $A$ - $\operatorname{Int} A$ is nowhere dense in $(A, \tau / A)$. Thus, if $D_{1} \cup D_{2}$ is a disjoint union of dense subsets of $\operatorname{Int} A$ then $D_{0}=D_{1} \cup(A-\operatorname{Int} A)$ and $D_{2}$ are disjoint and also are dense in $A$.

Lemma 1. (Corollary 5 of [1]) If $(X, \tau)$ is resolvable then $\tau_{P}=2^{X}$.

Proof. Let $D_{1}$ and $D_{2}$ be disjoint dense subsets of $X$ and let $x \in X$. Then $D_{1} \cup\{x\}$ and $D_{2} \cup\{x\}$ are dense and hence preopen. Thus $\{x\}=\left(D_{1} \cup\{x\}\right) \cap$ $\left.D_{2} \cup\{x\}\right) \in \tau_{P}$.

Lemma 2. $f:(X, \tau) \rightarrow(Y, \sigma)$ is SMPC iff $f:(X, \tau) \rightarrow\left(Y, \sigma_{P}\right)$ is continuous. 
Proof. A basic open set in $\sigma_{P}$ has the form $V=\bigcap_{k=1}^{n} B_{k}$ where each $B_{k} \epsilon$ $P O(Y, \sigma)$. So if $f:(X, \tau) \rightarrow(Y, \sigma)$ is SMPC, and $V$ is a basic open set in $\sigma_{P}$, $f^{-1}(V)=\bigcap_{k=1}^{n} f^{-1}\left(B_{k}\right) \in \tau$ so that $f:(X, \tau) \rightarrow\left(Y, \sigma_{P}\right)$ is continuous. The converse is clear since $P O(Y, \sigma) \subseteq \sigma_{P}$.

Theorem 2. If either (1) every open subset of $Y$ is closed, or $(2)(Y, \sigma)$ is resolvable then

$f:(X, \tau) \rightarrow(Y, \sigma)$ is SMPC iff $f:(X, \tau) \rightarrow\left(Y, 2^{Y}\right)$ is continuous.

Proof. By Lemmata 1 and 2 and the foregoing remarks, in either case, $\sigma_{P}=2^{Y}$.

We offer the following consequences.

Corollary 1. If $(Y, \sigma)$ is resolvable, the following are equivalent.

i. $f:(X, \tau) \rightarrow(Y, \sigma)$ is SMPC.

ii. $f^{-1}(B)$ is clopen (closed and open) for each $B \subseteq Y$.

iii. $f^{-1}(y)$ is clopen for each $y \in Y$.

iv. $f^{-1}(y)$ is open for each $y \in Y$.

v. $f:(X, \tau) \rightarrow\left(Y, 2^{Y}\right)$ is continuous.

Corollary 2. If $(X, \tau)$ is connected and $(Y, \sigma)$ is resolvable then $f:(X, \tau)$ $\rightarrow(Y, \sigma)$ is SMPC iff $f$ is a constant function.

For example if $R$ is the usual space of real numbers, every nonconstant function $f: R \rightarrow R$ is not SMPC.

Corollary 3. If $(X, \tau)$ is dense-in-itself (has no isolated points) and $(Y, \sigma)$ is a nonempty resolvable space then there is no SMPC injection $f:(X, \tau) \rightarrow$ $(Y, \sigma)$.

Theorem 3. If $(X, \tau)$ is a space, $X=X_{1} \cup X_{2}$ and $X_{1} \cap X_{2}=\phi$ and $X_{1}$ is closed then if both $\left(X_{1}, \tau / X_{1}\right)$ and $\left(X_{2}, \tau / X_{2}\right)$ are hereditarily irresolvable then $(X, \tau)$ is hereditarily irresolvable. 
Proof. Suppose that $\phi \neq A \subseteq X$ and $(A, \tau / A)$ is resolvable. Then there exist disjoint, dense in $A$, subsets $D_{1}$ and $D_{2}$ with $A=D_{1} \cup D_{2}$. Suppose that $D_{1} \cap X_{2} \neq \phi$, and $D_{2} \cap X_{2} \neq \phi$. Then since $X_{2}$ is open in $X, D_{1} \cap X_{2}$ and $D_{2} \cap X_{2}$ are disjoint and dense in $A \cap X_{2}$. For if $x \in D_{2} \cap X_{2}$ and $V$ is open with $x \in V$, since $D_{1}$ is dense in $A, V \cap A \cap D_{1} \neq \phi$. If $U$ is open in $X$ and $x \in U$ then, for $V=U \cap X_{2}, V \in \tau$ and $x \in V$ so that $U \cap A \cap\left(D_{1} \cap X_{2}\right) \neq \phi$. Thus, $D_{1} \cap X_{2}$ and similarly $D_{2} \cap X_{2}$ are dense in $A \cap X_{2}$ and disjoint. Thus, $A \cap X_{2}$ is a resolvable subspace of $X_{2}$ which contradicts $X_{2}$ being hereditarily irresolvable. Apparently, either $D_{1} \cap X_{2}=\phi$ or $D_{2} \cap X_{2}=\phi$. But in either case $A \cap X_{1}$ contains a dense set in $A$. Thus, $C l_{A}\left(A \cap X_{1}\right)=A \subseteq A \cap X_{1} \subseteq X_{1}$ since $X_{1}$ is closed. Thus $A$ is a resolvable subspace of $X_{1}$ which cannot be since $X_{1}$ is hereditarily irresolvable. This final contradiction proves that $(X, \tau)$ is hereditarily irresolvable.

We also note that every subspace of a hereditarily irresolvable space is hereditarily irresolvable.

\section{On Submaximality}

Proposition 1. For a submaximal space $(X, \tau)$, if $\rho$ is a finer topology than $\tau$ on $X$. Then $(X, \rho)$ is also submaximal.

Proof. If $D \subseteq X$ is $\rho$-dense then $X=C l_{\rho} D \subseteq C l_{\tau} D$ this leads to $D$ is $\tau$-dense and hence $D \in \tau$. Thus, $D \in \rho$ showing that $(X, \rho)$ is submaximal.

Theorem 4. Submaximality is preserved by open surjections.

Proof. If $f:(X, \tau) \rightarrow(Y, \sigma)$ is an open surjection and $(X, \tau)$ is submaximal and if $D \subseteq Y$ is dense, $f^{-1}(D)$ is dense and hence open in $X$ so that $D=$ $f\left(f^{-1}(D)\right)$ is open.

Corollary 4. If $\Pi X_{\alpha}$ is submaximal then each $X_{\alpha}$ is submaximal.

Now, we show that open and hence semi-open subsets of a submaximal space are submaximal. We first note the following useful known lemma. 
Lemma 3. If $A \in S O(X, \tau)$ then $\tau^{\alpha} / A=(\tau / A)^{\alpha}$.

Theorem 5. If. $(X, \tau)$ is submaximal and $A \in S O(X, \tau)$ then $(A, \tau / A)$ is submaximal.

Proof. Since $(X, \tau)$ is submaximal and $A S O(X, \tau)$. Then $\tau=\tau^{\alpha}$ and there is an open, dense, hereditarily irresolvable subset $D \subseteq X$. If $A \neq \phi$, then $D \cap \operatorname{Int} A$ is a dense, open, hereditarily irresolvable subspace of $(A, \tau / A)$, and also $C l_{A}(D \cap \operatorname{Int} A)=A \cap C l(D \cap \operatorname{Int} A)=A \cap C l \operatorname{Int} A=A$. Since $\tau / A=\tau^{\alpha} / A=$ $(\tau / A)^{\alpha}$, we have that $(A, \tau / A)$ is submaximal.

Lemma 4. (Proposition 1 of [1]) $A \in P O(X, \tau)$ iff $A=U \cap D$ for some $U \in \tau$ and dense $D \subseteq X$.

Proof. $A \in P O(X, \tau) \rightarrow A \subseteq \operatorname{Int} C l A=U \in \tau$. Let $D=X-(U-A)=$ $(X-U) \cup A$. Then $D$ is dense since $X=C l A \cup(X-C l A) \subseteq C l A \cup(X-U)=$ $C l D$. Also, $A=U \cap D$. Conversely, if $A=U \cap D$ with $U \in \tau$ and $D$ dense, $A \subseteq U \subseteq \operatorname{Int} C l U=\operatorname{Int} C l(A)$ so that $A \in P O(X, \tau)$.

Lemma 5. If $(X, \tau)$ is submaximal then $P O(X, \tau)=\tau$.

Proof. Clearly $\tau \subseteq P O(X, \tau)$. Now $A \in P O(X, \tau) \rightarrow A=U \cap D$ for some $U \in \tau$ and dense $D \subseteq X$. Therefore, if $(X, \tau)$ is submaximal, $D \in \tau \rightarrow A \in \tau$. Clearly the three parts of the next theorem follow from lemma 5.

Theorem 6. For $f:(X, \tau) \rightarrow(Y, \sigma)$, the following holds

(i) If $(X, \tau)$ is submaximal, then $f$ is SMPC iff it is preirresolute.

(ii) SMPC coincides with continuity if $(Y, \sigma)$ is submaximal.

(iii) If both $(X, \tau)$ and $(Y, \sigma)$ are submaximal, then $S M P C$, preirresolute precontinuity and continuity are equivalent.

\section{On SMPC}

Lemma 6. (Theorem 5 of [1]) For a space $(X, \tau)$ let $X=F \cup G$ denote the 
Hewitt-representation of $(X, \tau)$. Then $P O(X, \tau)$ is a topology on $X$ iff $C l G$ is open and $\{x\} \in P O(X, \tau)$ for each $x \in \operatorname{Int} F$.

Theorem 7. If a space $(Y, \sigma)$ as $(X, \tau)$ in Lemma 6 , then $f:(X, \tau) \rightarrow$ $(Y, \sigma)$ is SMPC iff $f:(X, \tau) \rightarrow(Y, P O(Y, \sigma))$ is continuous.

It was shown in Proposition 3.4 of [10] and independently in Theorem 1 of [7] that every precontinuous semi-open function is preirresolute, where a function is semi-open if images of open sets are semi-open. Consequently projections on product spaces are always preirresolute being both continuous and open. These suggests simpler proofs for next results in which we will abbreviate a space $(X, \tau)$ by $X$ and $\left\{X_{\alpha}: \alpha \in \nabla\right\}$ means the family of topological spaces.

Proposition 2. If $f: X \rightarrow \Pi X_{\alpha}$ is SMPC, then $p_{\alpha} f: X \rightarrow X_{\alpha}$ is SMPC, for each $\alpha \in \nabla$ (where $p_{\alpha}$ is the projection of $\Pi X_{\alpha}$ onto $X_{\alpha}$, for each $\alpha \in \nabla$ ).

Proof. Since $f: X \rightarrow \Pi X_{\alpha}$ is SMPC, then each $p_{\alpha}$ is preirresolute, each $p_{\alpha} \circ f$ is SMPC by Theorem 3.3 (v) (1) [9].

Corollary 5. Let $f_{\alpha}: X \rightarrow X_{\alpha}, \alpha \in \nabla$ be a class of functions defined as $f_{\alpha}(x)=x_{\alpha}$ and $f: X \rightarrow \Pi X_{\alpha}$ is given by $f(x)=\left\{f_{\alpha}(x)\right\}$ for each $x \in X$ and $\alpha \in \nabla$. If $f$ is SMPC then $f_{\alpha}$ is SMPC, for each $\alpha \in \nabla$.

Proof. By previous proposition, and the fact that each $f_{\alpha}=p_{\alpha} \circ f$.

Theorem 8. Each function of the family $f_{\alpha}: X_{\alpha} \rightarrow Y_{\alpha}, \alpha \in \nabla$ is SMPC if the function $f: \Pi X_{\alpha} \rightarrow \Pi Y_{\alpha}$, which is defined by $f\left\{x_{\alpha}\right\}=\left\{f_{\alpha}\left(x_{\alpha}\right)\right\}$ is SMPC.

Proof. Since $f$ is SMPC, then each $q_{\alpha} \circ f: \Pi X_{\alpha} \rightarrow Y_{\alpha}$ is SMPC by Proposition 2 where $q_{\alpha}: \Pi Y_{\alpha} \rightarrow Y_{\alpha}$ is the projection. Then if $p_{\alpha}: \Pi X_{\alpha} \rightarrow X_{\alpha}$, since $f_{\alpha} \circ p_{\alpha}=q_{\alpha}, f_{\alpha} \circ p_{\alpha}$ is SMPC. Now since $p_{\alpha}$ is open, by Theorem 3.3 (ii) $[9], f_{\alpha}$ is $\mathrm{SMPC}$.

The converse of Theorem 8 may not be hold in general, as the following example illustrates. 
Example 1. Let $X=\{0\} \cup\left\{\frac{1}{n}: n=1,2, \cdots\right\}$ have the usual real subspace topology. Then the only proper dense subset of $X$ is $D=\left\{\frac{1}{n}: n=1,2, \cdots\right\}$ which is open so that $X$ is submaximal. By Lemma 5 above or Theorem 3.1 [9], the identity function $1_{X}: X \rightarrow X$ is SMPC. However, $1_{X} \times 1_{X}=1_{X}^{2}$ : $X^{2} \rightarrow X^{2}$ is the identity function on the product space $X^{2}$ and is not SMPC. For $\{(0,0)\} \cup(D \times D)$ is dense and hence preopen in $X^{2}$ but not open. Consequently, also, $X^{2}$ is not submaximal. However, $X^{2}$ is hereditarily irresolvable for if $Y_{1}=$ $\{0\} \times X \cup X \times\{0\}$ and $Y_{2}=X^{2}-Y_{1}$, then it is easily seen that as subspaces of $X^{2}, Y_{1}$ and $Y_{2}$ are each hereditarily irresolvable and further $Y_{1}$ is closed in $X^{2}$. The result follows from Theorem 3 .

\section{References}

[1] M. Ganster, "Preopen sets and resolvable spaces", to appear in Kyungpook Math. J.

[2] E. Hewitt, "A problem of set theoretic topology", Duke Math. J. 10 (1943), 309-333.

[3] D. E. Cameron, "A Class of maximal topologies", Pacific J. of Math., 7 (1977), 101-104.

[4] A. S. Mashhour, M. E. Abd El-Monsef and S. N. El-Deeb, "On precontinuous and weakprecontinuous mappings", Proc. Math. and Phys. Soc. Egypt, 53 (1982), 47-53.

[5] A. S. Mashhour, I. A. Hasanein and S. N. El-Deeb, " $\alpha$-continuous and $\alpha$-open mappings", Acta Math. Acad. Sci. Hungar, 41 (1983), 213-218.

[6] N. Levine, "Semi-open sets and semi-continuity in topological spaces", Amer. Math. Monthly, 70 (1963), 36-41. [7] A. D. Rose, "Subweakly $\alpha$-continuous functions", Inter. J. of Math. and Math. Sci., 11
(1988), 713-720.

[8] I. L. Reilly and M. K. Vamanamurthy, "On $\alpha$-continuity in topological spaces", Acta Math. Hungar, 45 (1985), 27-32.

[9] R. A. Mahmoud, M. E. Abd E1-Monsef and A. A. Nasef, "A Class of functions stroger than $M$-precontinuous, preirresolute and $A$-function", to appear in Qatar Univ. Sci. Bull.

[10] D. S. Jankovic, "A Note on mappings of extremally disconnected spaces", Acta Math. Hungar, 46 (1985), 83-92. [11] S. G. Grossely, and S. K. Hildebrand, "Semi-topological properties", Fund. Math., 74
(1972), 233-254.

Department of Mathematics, Faculty of Science, Menoufia University, Egypt.

Department of Mathematics, East Central University, Oda, Oklahoma, 74820, U. S. A. 